1 Universidade do Estado de Santa Catarina (Udesc) - Chapecó (SC), Brasil. Iferraz@unochapeco.edu.br

2 Universidade Comunitária da Região de Chapecó (Unochapecó) - Chapecó (SC), Brasil.

3 Universidade do Minho Braga, Portugal.

4 Universidade do Porto Porto, Portugal.

\section{Tradução do Conhecimento e os desafios contemporâneos na área da saúde: uma revisão de escopo}

\author{
Knowledge Translation and contemporary challenges in the health \\ field: a scope review
}

Lucimare Ferraz $\mathbf{1 , 2}$, Rui Pedro Gomes Pereira $\mathbf{3}$, Altamiro Manuel Rodrigues da Costa Pereira $\mathbf{4}$

DOI: 10.1590/0103-11042019S215

RESUMO A implementação das melhores evidências científicas nos serviços de saúde ainda não ocorre de forma satisfatória. Diante dessa problemática, o objetivo deste estudo foi investigar os desafios da Tradução do Conhecimento (TC) na área da saúde na atualidade. A metodologia desta revisão foi desenvolvida de acordo com os propósitos da revisão de escopo. Para tanto, as palavras-chave 'translational medical research' e 'knowledge translation' foram consultadas nos bancos de dados de periódicos da PubMed, Scopus e Web of Science. Foram incluídos os estudos publicados a partir do ano de 2008 até abril de 2018. Entre os 1.677 estudos encontrados, 839 artigos eram duplicados, e 818 não atendiam plenamente ao objetivo desta revisão; assim, 20 estudos foram submetidos à apreciação desse escopo. De acordo com as análises dos estudos, o desafio da TC advém de dois fatores: por um lado, a falta de coesão entre a comunidade científica e os tomadores de decisão em saúde; por outro, a inabilidade dos profissionais em traduzir e aplicar novos conhecimentos, além da omissão de apoio e de incentivos das instituições de saúde. Outrossim, esta revisão aborda um corpo significativo de diversos outros aspectos que limitam e/ou dificultam a TC área da saúde.

PALAVRAS-CHAVE Pesquisa médica translacional. Serviços de saúde. Pessoal de saúde. Prática clínica baseada em evidências.

\begin{abstract}
The implementation of the best scientific evidence in health services has not yet occurred in a satisfactory way. Faced with this problem, the objective of this study was to investigate today's challenges of Knowledge Translation in the health field. The methodology of this review was developed according to the requirements of the scope review. Therefore, the keywords 'translational medical research' and 'knowledge translation' were consulted in the databases of PubMed, Scopus, and Web of Science journals. Studies published from 2008 to April 2018 were included. Among the 1,677 studies found, 839 articles were duplicated and 818 did not fully meet the objective of this review, therefore, 20 studies were submitted to review and critical appraisal. According to the analysis of the studies, the challenge of Knowledge Translation comes from two factors: on the one hand, the lack of cohesion between the scientific community and health decision makers and, on the other hand, the inability of professionals to translate and apply new knowledge, in addition to the lack of support and incentives from health institutions. Furthermore, this review addresses a significant body of several other aspects that limit and/or hinder the translation of knowledge in the health field.
\end{abstract}

KEYWORDS Translational medical research. Health services. Health personnel. Evidence-based practice. 


\section{Introdução}

A Tradução do Conhecimento (TC) é definida como síntese, intercâmbio e aplicação de novos saberes por pessoas interessadas em acelerar os benefícios da inovação global e local, fortalecendo os sistemas de saúde e a melhoria da saúde da população1. O Canadian Institutes of Health Research (CIHR), criado em 2000, define a TC como um processo dinâmico e interativo, que inclui a síntese, a disseminação, o intercâmbio e a aplicação ética do conhecimento para melhorar a saúde, por meio de serviços e produtos mais efetivos, fortalecendo os sistemas de saúde².

A Organização Mundial da Saúde (OMS) adotou a definição CIHR de TC adaptando-a para a síntese, o intercâmbio e a aplicação do conhecimento pelas partes interessadas para acelerar os benefícios da inovação global e local dos sistemas de saúde e a melhoria da saúde das pessoas ${ }^{3}$.

Nessa perspectiva, a TC abrange todas as fases entre a criação de novos conhecimentos e sua aplicação para produzir resultados benéficos para a sociedade. Isso abarca a disseminação, a comunicação, a transferência de tecnologia, o contexto ético, o gerenciamento, a utilização, o intercâmbio de informações entre pesquisadores e aqueles que aplicam conhecimento, pesquisa de implementação e avaliação de tecnologia, a síntese de resultados em um contexto global e o desenvolvimento de diretrizes (CIHR) ${ }^{2}$.

Além da síntese e da aplicação ética do conhecimento, por meio de um sistema complexo de interação entre pesquisadores e profissionais de saúde, a trasladação do conhecimento necessita de uma comunicação efetiva sobre resultados de pesquisa, bem como a revisão, a identificação e a aplicação prática de estudos pelos stakeholders (pessoas ou instituições interessadas, que têm um papel importante na gestão dos resultados de pesquisa) ${ }^{4}$.

O objetivo da TC é colocar em prática a melhor evidência disponível para os serviços de saúde, quer seja para planejar os serviços de saúde, averiguar o tratamento mais efetivo para as doenças ou orientar os profissionais de saúde na tomada de decisão no momento do atendimento. No entanto, abordagens para TC ainda estão sendo desenvolvidas, e os mecanismos pelos quais a TC melhor ocorre não são totalmente claros, particularmente quando a natureza do conhecimento a ser traduzido e os contextos para a absorção de conhecimento variam ${ }^{5}$.

Outrossim, o volume de conhecimento de saúde baseado em evidências disponível, e a velocidade em que é gerado, supera a capacidade contemporânea de divulgação, adoção e aplicação na prática clínica ${ }^{6}$. Embora haja uma robusta produção de conhecimento, ainda há múltiplos desafios para mover os resultados da pesquisa além do escopo de um projeto, influenciando efetivamente nos cuidados de saúde, uma vez que a ciência da implementação tem algum caminho a percorrer para que evidências científicas impactem nos serviços de saúde, de forma a garantir aos pacientes que a sua saúde esteja em boas mãos 7 .

Apesar da TC ser (re)conhecida como imperativa para os avanços na área da saúde e para as transformações sociais, isso ainda é um processo complexo ${ }^{8}$. Existem, em diferentes contextos, fatores que apoiam ou trabalham contra a trasladação do conhecimento, que é multidimensional e exige a interação entre os diferentes atores envolvidos em seu processo ${ }^{4}$.

Perante esse contexto, desenvolveu-se um estudo de revisão de escopo com o objetivo de investigar os desafios da TC na área da saúde na atualidade.

\section{Metodologia}

Esta revisão foi elaborada de acordo com metodologia de um scoping review (análise de escopo) recomendada pelo Instituto Joanna Briggs 9 . A técnica de scoping review está sendo amplamente utilizada na área das ciências da saúde com a finalidade de sintetizar e de disseminar os resultados de estudos a respeito de um assunto ${ }^{10-13}$. O objetivo de uma análise 
de escopo é mapear, por meio de um método rigoroso e transparente, o estado da arte em uma área temática, pretendendo fornecer uma visão descritiva dos estudos revisados, sem avaliá-los criticamente ou sumarizar evidências de diferentes investigações, como ocorre em uma revisão sistemática ${ }^{14}$.

As revisões do escopo diferem das revisões sistemáticas, porque não visam avaliar a qualidade das evidências disponíveis, mas objetivam mapear rapidamente os principais conceitos que sustentam uma área de pesquisa ${ }^{\mathbf{1 4}}$. Por outro lado, elas diferem de uma revisão tradicional da literatura na medida em que envolvem um procedimento mais sistemático.

Nessa perspectiva, esta revisão de escopo usou o arcabouço metodológico proposto por Arksey e O'Malley ${ }^{14} \mathrm{com}$ as emendas feitas por Levac, Colquhoun e O'Brien ${ }^{10}$ e por Peters e colaboradores ${ }^{15}$, estudiosos do Instituto Joanna Briggs. Adaptando-se aos nossos propósitos, a estrutura desta revisão consiste em seis principais etapas consecutivas: 1) identificação da questão e objetivo de pesquisa; 2) identificação de estudos relevantes, que viabilizassem a amplitude e abrangência dos propósitos da revisão; 3) seleção de estudo, conforme os critérios predefinidos; 4) mapeamento de dados; 5) sumarização dos resultados, por meio de uma análise temática qualitativa em relação ao objetivo e pergunta; 6) apresentação dos resultados, identificando as implicações para política, prática ou pesquisa;

Inicialmente, foi definida a seguinte pergunta de investigação: quais os desafios da TC na área da saúde na atualidade? Na sequência, após a elaboração da pergunta, foram identificadas as palavras-chave que conseguissem captar os artigos referentes à temática desta pesquisa, a saber: 'translational medical research' [Medical Subject Headings - MeSH
Terms] e 'knowledge translation'.

Para a identificação dos estudos relevantes, foram consultados os bancos de dados de periódicos da PubMed, Scopus e Web of Science. Essas bases de dados foram selecionadas por serem abrangentes, tendo ampla cobertura das publicações na área da saúde. Utilizou-se a seguinte estratégia de busca na Knowledge Translation [Title] OR translational Medical Research [Title/Abstract]. A estratégia de busca foi desenvolvida pelo primeiro autor (pesquisador principal) em colaboração com um bibliotecário da Faculdade de Medicina da Universidade do Porto.

A captura dos documentos restringiu-se ao seguinte critério de inclusão: artigos publicados em periódicos indexados na área da saúde. $\mathrm{O}$ ano inicial para o começo da busca foi 2008 , sendo que a coleta (e atualização) dos estudos ocorreu no dia 18 abril de 2018.

Todos os 1.677 estudos encontrados foram incorporados no software EndNote. Na sequência, foi realizada a identificação e a exclusão dos 839 artigos duplicados, restando 838 estudos. Destes, após leitura dos títulos e resumos, 773 foram excluídos por não apresentar elementos que atendessem ao objetivo desta revisão. Ressalta-se aqui que, quando a relevância de um estudo não era clara a partir do resumo, o artigo completo era recuperado para a sua leitura na íntegra pelo primeiro autor para verificar se eles abordavam adequadamente a questão de pesquisa. Os 65 que permaneceram na seleção foram lidos na íntegra; destes, 45 foram excluídos. As razões mais comuns para a exclusão dos estudos foi por não abordarem de forma substancial os desafios da TC na área da saúde. A figura 1 apresenta, como base na recomendação Prisma ${ }^{16}$, o fluxograma do processo de seleção das publicações desta revisão. 
Figura 1. Diagrama de fluxo de identificação, triagem e inclusão de estudos

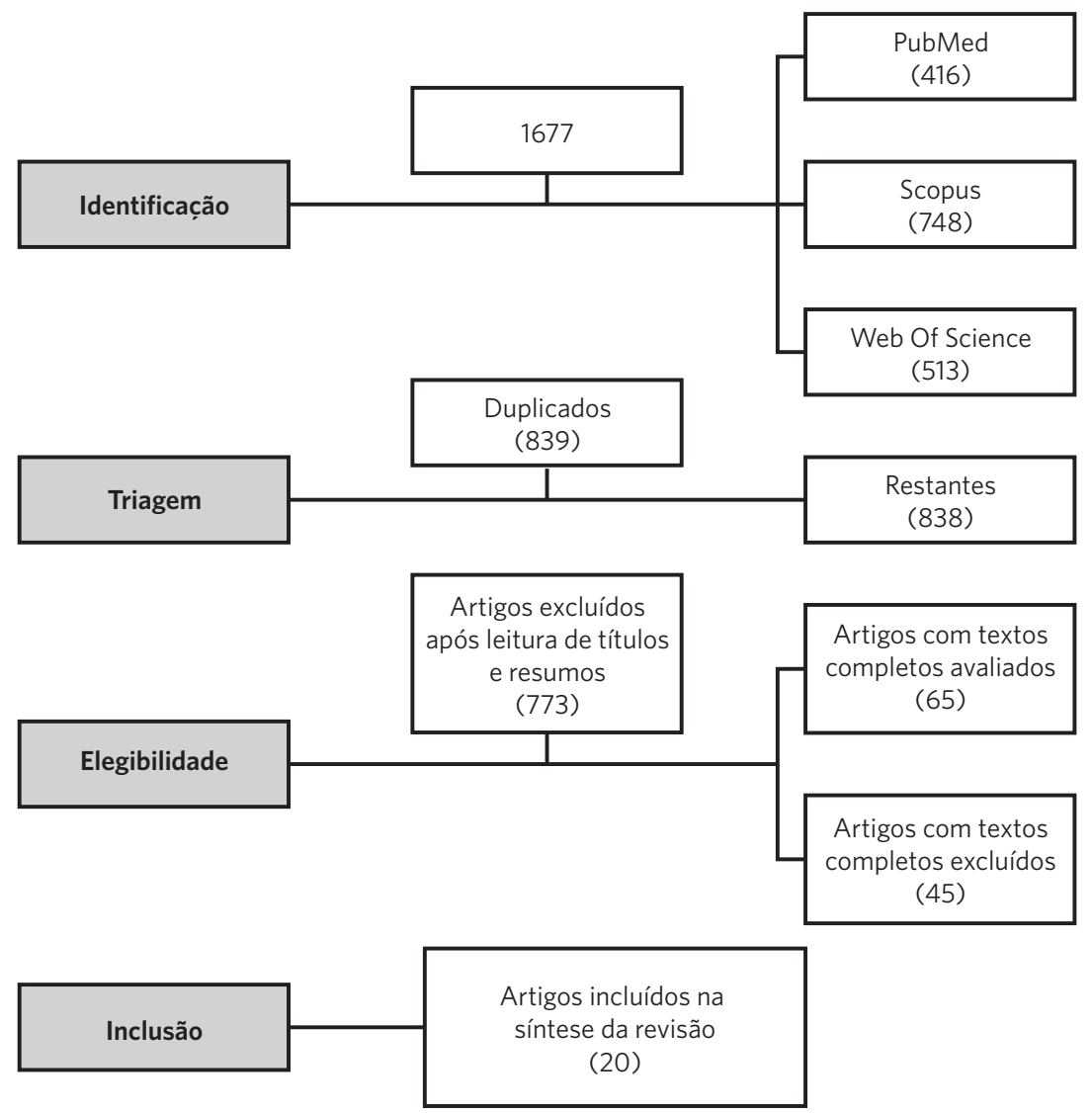

Fonte: Elaboração própria.

Os 20 estudos selecionados para fazer parte desta revisão foram mapeados por meio de uma planilha no programa Excel ${ }^{\circledR}$ com as seguintes informações: autor(es), ano de publicação, título, país de origem e trechos descrevendo os principais resultados de interesse desta revisão.

Para a etapa de sumarização dos elementos essenciais de cada estudo, utilizou-se uma estrutura analítica descritiva para examinar o texto de cada artigo. Para tanto, realizou-se uma análise qualitativa de todos os conteúdos, o que possibilitou a criação de categorias que emergiram da análise mais aprofundada das publicações, as quais foram capazes de ilustrar de tópicos de interesse.

Na etapa final, realizaram-se a compilação e a comunicação dos resultados, com a intenção de apresentar a visão geral de todo o material, por meio de uma construção temática, organizada de acordo com os elementos que influenciam na resposta da questão desta investigação. Na apresentação dos resultados desta revisão, construíram-se resumos narrativos, tratando-se de um processo.

\section{Resultados}

Constatou-se, nos estudos analisados (quadro 1), que a TC tem diversos desafios a serem superados, os quais foram sintetizados nesta revisão de escopo em duas grandes categorias: Falta de coesão entre a comunidade científica e os tomadores de decisão em saúde; Deficiência de habilidades e recursos para a Tradução conhecimento. 
Quadro 1. Estudos incluídos na revisão de escopo sobre os desafios da Tradução do Conhecimento, 2018

\begin{tabular}{|c|c|c|}
\hline Ano & Título & Autor(es) ${ }^{\star}$ \\
\hline 2008 & Poor knowledge translation: an urgent problem in China. & Hu G.; Sun Z. \\
\hline 2010 & $\begin{array}{l}\text { Analyzing the barriers and supports of knowledge translation using the } \\
\text { PEO model. }\end{array}$ & Metzler MJ.; Metz GA. \\
\hline 2010 & $\begin{array}{l}\text { Knowledge translation in emergency medical services: a qualitative } \\
\text { survey of barriers to guideline implementation. }\end{array}$ & Bigham BL. (et al.) \\
\hline 2011 & $\begin{array}{l}\text { Knowledge translation research: the science of moving research into } \\
\text { policy and practice. }\end{array}$ & Curran JA. (et al.) \\
\hline 2012 & Advancing knowledge translation in primary care. & Menear M. (et al.) \\
\hline 2012 & $\begin{array}{l}\text { Barriers to Knowledge Production, Knowledge Translation, and Urban } \\
\text { Health Policy Change: Ideological, Economic, and Political Considera- } \\
\text { tions. }\end{array}$ & Muntaner C. (et al.) \\
\hline 2012 & $\begin{array}{l}\text { Creating a Knowledge Translation Platform: nine lessons from the Zam- } \\
\text { bia Forum for Health Research. }\end{array}$ & Kasonde JM.; Campbell S. \\
\hline 2012 & $\begin{array}{l}\text { Taking power, politics, and policy problems seriously: the limits of know- } \\
\text { ledge translation for urban health research. }\end{array}$ & Murphy K.; Fafard P. \\
\hline 2013 & $\begin{array}{l}\text { Perspectives on the role of stakeholders in knowledge translation in } \\
\text { health policy development in Uganda. }\end{array}$ & Nabyonga Orem J. (et al.) \\
\hline 2014 & $\begin{array}{l}\text { Beyond two communities - from research utilization and knowledge } \\
\text { translation to co-production? }\end{array}$ & Wehrens R. (et al.) \\
\hline 2014 & $\begin{array}{l}\text { Differences in evaluating health technology assessment knowledge } \\
\text { translation by researchers and policymakers in China. }\end{array}$ & Liu W. (et al.) \\
\hline 2014 & $\begin{array}{l}\text { Exploring conceptualizations of knowledge translation, transfer and } \\
\text { exchange across public health in one UK region: a qualitative mapping } \\
\text { study. }\end{array}$ & Visram S.; Goodall D.; Steven A. \\
\hline 2014 & Research's Practice and Barriers of Knowledge Translation in Iran. & Nedjat S. (et al.) \\
\hline 2015 & $\begin{array}{l}\text { Exploring the Hidden Barriers in Knowledge Translation: A Case Study } \\
\text { Within an Academic Community. }\end{array}$ & Harvey G. (et al.) \\
\hline 2015 & Knowledge translation status and barriers. & Azimi A.; Fattahi R.; Asadi-Lari M. \\
\hline 2015 & Knowledge translation: translating research into policy and practice. & $\begin{array}{l}\text { Oelke ND.; da Silva Lima MA.; } \\
\text { Acosta AM. }\end{array}$ \\
\hline 2015 & $\begin{array}{l}\text { Swiss family physicians' perceptions and attitudes towards knowledge } \\
\text { translation practices. }\end{array}$ & Bengough T. (et al.) \\
\hline 2016 & Physicians and knowledge translation of statistics: Mind the gap. & Bartlett G.; Gagnon J. \\
\hline 2017 & Expanding the knowledge translation metaphor. & $\begin{array}{l}\text { Engebretsen E.; Sandset T. J.; } \\
\text { Odemark J. }\end{array}$ \\
\hline 2018 & $\begin{array}{l}\text { Determinants of knowledge translation from health technology assess- } \\
\text { ment to policy-making in China: From the perspective of researchers. }\end{array}$ & Liu W. (et al.) \\
\hline
\end{tabular}

Fonte: Elaboração própria.

*Estudo com mais de três autores, somente o primeiro autor foi mencionado.

De modo geral, os estudos apontam que existem lacunas entre os universos de produção e de consumo do conhecimento. Isso ocorre porque não há uma interação efetiva entre as partes no momento de elaboração dos projetos de pesquisa, tampouco no planejamento das estratégias de implementação de novos conhecimentos na assistência à saúde, como demonstrado na figura 2 . 
Figura 2. Apresentação dos núcleos, e seus distanciamentos, no processo da Tradução do Conhecimento

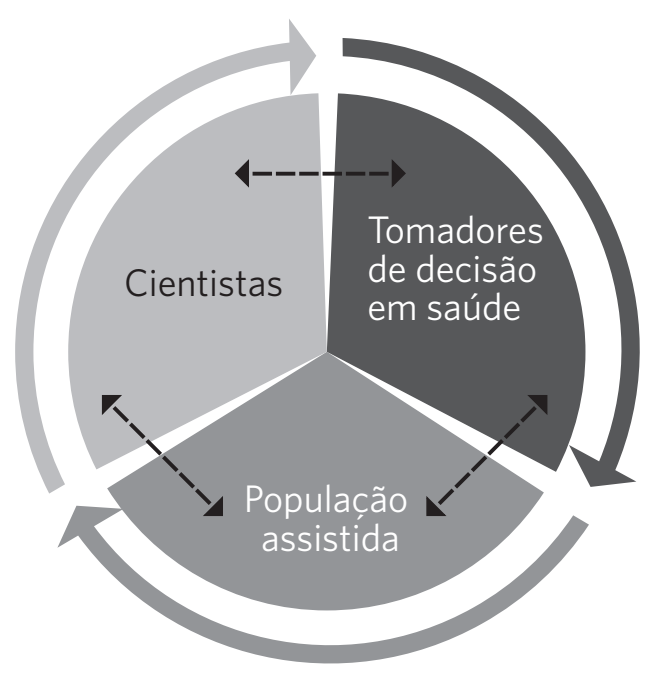

Fonte: Elaboração própria.

\section{Falta de coesão entre a comunidade científica e os tomadores de decisão em saúde}

O distanciamento entre comunidades de pesquisa e de saúde é visto como um grande entrave à TC, pois o compartilhamento de informações por meio de relacionamentos entre as partes é uma ferramenta potencialmente poderosa no arsenal de $\mathrm{TC}^{17}$. A falta de parceria entre pesquisadores e tomadores de decisão no processo de investigação é uma lacuna entre a pesquisa e a prática ${ }^{18}$, sendo que a falha em não abordar as prioridades da comunidade, e, sim, de forma desproporcional, os interesses acadêmicos ${ }^{19}$, ainda é um desafio a ser superado segundo os estudos desta revisão. Para que a prática de TC seja mais eficaz, a pesquisa precisa ser mais responsiva às necessidades de conhecimento dos médicos e outros provedores de cuidados ${ }^{\mathbf{2 0}, 21}$.

Entre os desafios, está o de desconstruir os valores e crenças que constituem a pesquisa como convencionalmente realizada na academia. Por isso, a pesquisa seria isenta de valor; e seus pesquisadores, desapegados ${ }^{22}$. Nesse sentido, refletem-se fraquezas na universidade em não determinar prioridades de pesquisa por intermédio de reuniões com partes interessadas, com comunicação entre pesquisadores e público-alvo da pesquisa, bem como o acompanhamento, o suporte, os regulamentos e as medidas da utilização dos resultados da pesquisa para a $\mathrm{TC}^{\mathbf{2 3}}$. Outra questão que leva ao enfraquecimento da TC é a escolha por parte do pesquisador de tópicos (temas) de investigação com base no escopo de revistas, e isso acaba por afastar o pesquisador das necessidades de pesquisa da comunidade ${ }^{24}$.

Por conseguinte, pesquisadores devem começar a reavaliar se o estudo proposto irá contribuir não somente para o desenvolvimento de novo conhecimento, mas também para traduzir esse conhecimento para a prática. Por isso, precisam estar conscientes dos interesses e das necessidades dos profissionais de saúde, tomadores de decisão e formuladores de políticas $^{18}$. A natureza acadêmica da pesquisa pode estar negativamente associada ao nível de TC, quando não houver significativos vínculos entre pesquisadores e formuladores de políticas ${ }^{25}$.

Uma percepção de que pesquisadores, profissionais e formuladores de políticas derivam de mundos estritamente separados, 
com lógicas, motivações e incentivos distintos, é frequentemente descrita como lacunas da $\mathrm{TC}^{26}$. Apesar da comunidade política, por muitas vezes, formular diretrizes e/ou políticas sem consultar evidências de pesquisa - tendo poucas conexões ativas com pesquisadores independentes ou com centros de pesquisa -, é preciso alinhar tópicos de investigação com necessidades políticas de saúde, indo além das necessidades ou desejos dos financiadores, pois a TC é, acima de tudo, um processo social27. Nesse acaso, a TC convencional silencia sobre o papel do poder e da política na formulação de políticas públicas; e como consequência, as estratégias de TC podem ser difíceis de executar28, ainda mais quando há particularidades conflitantes entre as partes interessadas na tradução, transferência e intercâmbio de conhecimento em saúde pública ${ }^{29}$.

Essa desconexão força os provedores de cuidados a avaliar a aplicabilidade dos achados e intervenções de pesquisa aos pacientes do mundo real em seus próprios contextos de prática, um processo desafiador de moldar a pegada quadrada da evidência para se ajustar ao buraco redondo da vida do paciente ${ }^{20}$. No entanto, é crucial adaptar o conhecimento para o contexto local, avaliar barreiras e facilitadores para seu uso e dirigi-lo para as necessidades das pessoas que irão usá-1o ${ }^{18}$.

Outro importante desafio que foi identificado para a TC é a falta de diálogo entre os pesquisadores e formuladores de políticas ${ }^{24-26}$. Há diferenças culturais entre os domínios de pesquisa, política e prática ${ }^{26}$. No entanto, a TC é, acima de tudo, um processo social, implicando a construção de confiança e diálogo entre pesquisadores e formuladores de políticas, sendo necessário alinhar tópicos de pesquisa com necessidades políticas ${ }^{27}$.

A complexidade do processo de formulação de políticas requer o abandono da noção de decisão única, pois as decisões políticas ocorrem, por definição, dentro de um campo de poder social, econômico e político ${ }^{28}$. Portanto, é necessária uma interrogação das causas políticas no escopo das investigações, considerando as relações de classe política e social que reproduzem os determinantes sociais de saúde ${ }^{22}$.

É possível que os formuladores de políticas não se oponham tanto ao rigor científico da pesquisa como alienados por tecnicismos impenetráveis e por jargões acadêmicos em alguns relatórios ${ }^{25}$. Entretanto, os tomadores de decisão política consideraram a natureza excessivamente pedante da pesquisa científica como um obstáculo para a $\mathrm{TC}^{30}$. Esta, por sua vez, não pode ser fortalecida somente pela realização de intervenções apenas no lado do empurrão (por parte dos pesquisadores) e sem a cooperação de formuladores de políticas nos níveis macro e médio ${ }^{24}$. Por outro lado, há a ressalva de que os tomadores de decisão política são inclinados, em primeiro lugar, para servir interesses políticos ${ }^{\mathbf{1 9}}$.

Assim, as relações de poder têm efeitos diretos e indiretos sobre as formas como as evidências de pesquisa são recebidas, percebidas e mobilizadas no processo de desenvolvimento de políticas, e elas precisam ser levadas em consideração ao planejar e implementar uma estratégia de TC para a saúde urbana ${ }^{28}$.

Não obstante que os pesquisadores estejam conscientes dos interesses e necessidades dos profissionais de saúde, tomadores de decisão e formuladores de políticas ${ }^{18}$, a comunicação deficiente - pela falta de praticidade dos relatórios de pesquisa que apresentem evidências em linguagem de fácil compreensão - é uma limitação da $\mathrm{TC}^{\mathbf{3 0}, 31}$.

Traduzir os resultados de pesquisa em elaboração de políticas é um ato de equilíbrio difícil entre garantir a relevância da pesquisa e de preservar a integridade acadêmica, porém, apesar da necessidade de manter o rigor científico na pesquisa, é igualmente importante assegurar que a sua disseminação seja feita de maneira amigável25. Considerando que a interpretação estatística é difícil, sendo uma barreira para a TC, linguagens e exibições visuais mais acessíveis podem ser necessárias para tornar os resultados estatísticos de meta-análises mais compreensíveis por exemplo ${ }^{31}$. 
No entanto, há o desafio de implementar a mensagem científica original em novos contextos sociais sem alterar seu conteúdo, e os modelos de TC existentes não conseguem transpor esse paradoxo - a tradução é inerente à ciência, e a divisão entre a ciência e sua tradução é impossível e improdutiva de se manter ${ }^{32}$.

Além disso, relatórios pobres são obstáculos para a TC, pois resultam em uma visão limitada da lógica e especificação da intervenção, sendo que uma abrangente avaliação, incluindo informações sobre os processos, mecanismos de ação e análise econômica de implementação de novas práticas, faz-se necessária ${ }^{33}$. Se por um lado os relatórios precisam estar escritos de maneira compreensível24, por outro, os pesquisadores que trabalham no campo de TC, além de prestar especial atenção ao uso da linguagem, necessitam reportar descrições ricas sobre o contexto e as estratégias de implementação, buscando explicitar as razões de sucesso ou fracasso das intervenções ${ }^{33}$. A organização e a apresentação dos resultados de pesquisa são um estágio essencial da TC, porém muitas vezes negligenciado pelos cientistas da área da saúde, que se dedicam à pesquisa com pouca atenção às formas nas quais o conhecimento deve ser organizado antes de ser implementado na vida real21.

\section{Deficiência de habilidades e recursos para a Tradução do Conhecimento}

Os estudos também apontam que a ausência de familiaridade com a TC pelos pesquisadores - verificada na falta de consciência de seus conceitos, ferramentas e necessidades - é um desafio importante a ser transpassado ${ }^{24}$. Do mesmo modo, no nível semântico, existem diferentes significados ligados ao conceito de $\mathrm{TC}$, sendo que há questões relativas também no nível sintático, no qual a linguagem e a terminologia da TC são familiares para alguns, mas não para outros ${ }^{34}$.

Faz-se necessário criar, portanto, uma gama mais ampla de metáforas e modelos de TC que levem em conta que a formulação de políticas é um processo que envolve muito mais etapas do que a simples implementação de soluções baseadas em evidências ${ }^{28}$. É imperativo conceber um modelo expandido de TC que incorpore uma concepção mais dinâmica de tradução e uma descrição mais adequada e reflexiva da interdependência de práticas científicas, culturais, textuais e materiais, facilitando a integração das evidências de pesquisa e do conhecimento desenvolvido no domínio da prática, incluindo o conhecimento do paciente e a experiência clínica ${ }^{32}$.

Nesta revisão, verifica-se que a forma como a pesquisa acadêmica é financiada e incentivada é vista como uma forte barreira à $\mathrm{TC}^{29}$. A deficiência de recursos financeiros para a transferência de conhecimento ${ }^{23}$, e/ ou financiamento adequado para pesquisa, faz com que haja uma capacidade limitada para desenvolver e implementar um plano de $\mathrm{TC}^{18,25}$. Uma consideração cuidadosa da sustentabilidade da TC, no que se refere aos efeitos das intervenções ao longo do tempo, é o financiamento de pesquisa ${ }^{33}$, uma vez que eles se limitam somente aos custos da produção do conhecimento.

Vale destacar que as fundações privadas e os doadores são fontes cada vez mais importantes de financiamento da pesquisa em saúde global, o que tem um enorme impacto na definição de quais problemas de saúde são considerados prioritários e quais soluções são recomendadas, visto que os pesquisadores tendem a elaborar propostas com perguntas e métodos de pesquisa que correspondam aos interesses dos financiadores ${ }^{\mathbf{2 2}}$. No entanto, desafios relacionados com incentivos e recompensas para a TC provavelmente exigirão discussões mais sustentadas e negociadas com uma variedade de partes interessadas dentro do contexto mais amplo da universidade e das políticas acadêmicas ${ }^{\mathbf{3 4}}$.

Outro desafio bastante destacado nos estudos é a inabilidade dos profissionais de saúde em buscar, selecionar e implementar a TC em suas práticas ${ }^{10,18,31,35}$. Os profissionais de saúde enfrentam barreiras ao tentar acessar 
informações por falta de habilidade em pesquisa ${ }^{17,20}$. Entre as dificuldades, destaca-se o pouco domínio da estatística, que está repleta de conceitos e termos técnicos que podem ser difíceis de interpretar, e isso representa uma barreira importante para o uso do conhecimento ${ }^{31}$. Conhecimentos e habilidades insuficientes dos profissionais de saúde sobre a avaliação crítica das informações científicas representam uma barreira à $\mathrm{TC}$, uma vez que não se sentem capazes de determinar plenamente se as informações apresentadas em artigos de pesquisa são válidas ou como resultados de ensaios clínicos são confiáveis, imparciais e viáveis de implementação ${ }^{35}$.

De acordo com os estudos, há falta de apoio nos ambientes institucionais para TC. Não há, de forma satisfatória, nos serviços de saúde treinamento para que os profissionais de saúde se capacitem quanto à busca, seleção e implementação de melhores evidências científicas em suas práticas clínicas ${ }^{\mathbf{1 7}, 24,36}$. Outro aspecto institucional é o tempo limitado que os profissionais de saúde dispõem para se dedicar ao acompanhamento das publicações científicas e se manter atualizados ${ }^{35}$. Isso também ocorre porque as bases de evidências de pesquisa são extensas e estão em constante fluxo, dificultando para que os profissionais se mantenham informados acerca dos avanços científicos, sendo essa dificuldade agravada pelo tempo limitado que têm nas instituições de saúde para essa atividade ${ }^{29}$.

A pouca infraestrutura disponibilizada nas instituições também é outro fator que desfa-

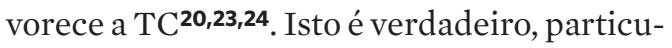
larmente em termos de dificuldade de acesso a recursos e tecnologias ${ }^{\mathbf{1 7}}$ que disponibilizam informações. A ausência de um mecanismo predefinido para transferir descobertas de pesquisas para públicos-alvo é uma barreira importante. Por isso, a questão da infraestrutura exige atenção especial24. Além da limitação de infraestrutura, há nas instituições uma demora em aplicar novos conhecimentos ${ }^{19,36}$. Nesse caso, além do atraso na tomada de decisões pelos reguladores do governo em aprovar novas diretrizes, há também uma leniência dos gestores locais, como diretores médicos e hospitalares, no que concerne à implementação de novos resultados de pesquisa ${ }^{36}$. Desse modo, o desafio encontra-se em aproximar e integrar todos os elementos desse processo, como apresenta a figura 3.

Figura 3. Apresentação verticalizada e integrada dos elementos da Tradução do Conhecimento

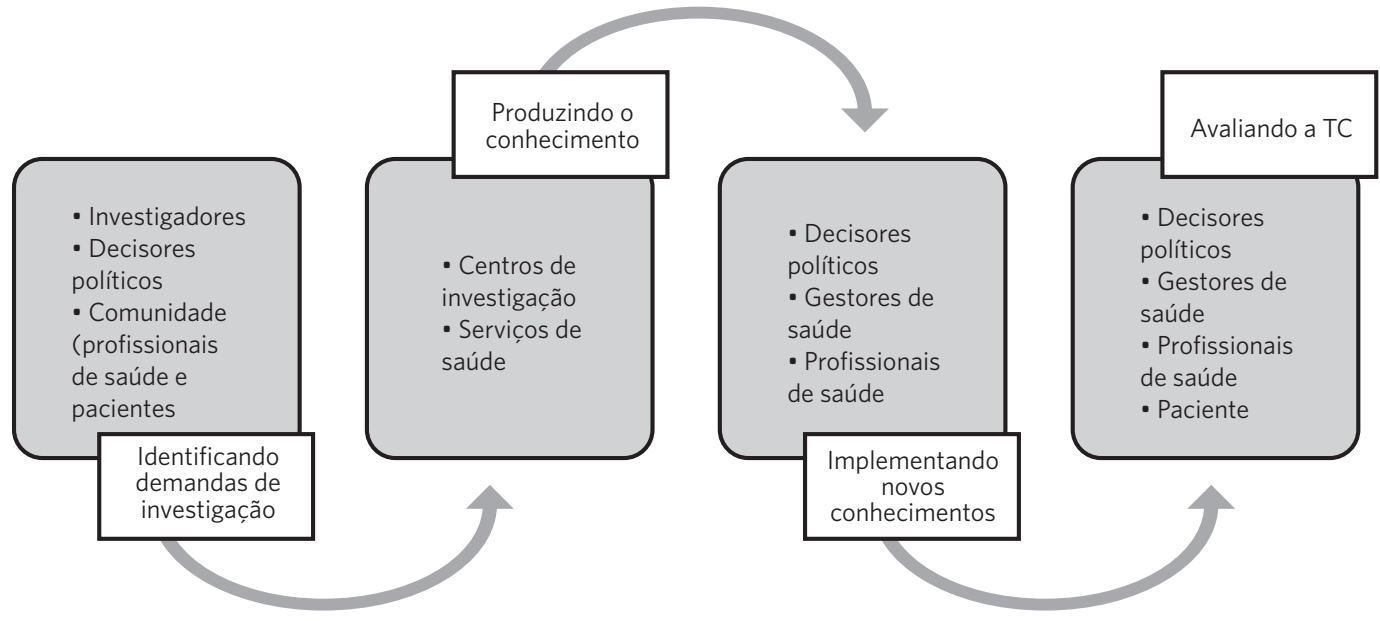




\section{Discussões}

Esta revisão de escopo identifica uma série de desafios para o desenvolvimento da TC na área da saúde. A maioria dos estudos incluiu a falta de conexão entre pesquisadores (produtores de novos conhecimentos) e comunidade (de consumo desses conhecimentos) como a principal barreira da TC. Contudo, os estudos apresentam um corpo significativo de diversos outros aspectos que limitam e/ou dificultam a TC, como a falta de apoio institucional; a inabilidade em buscar e selecionar evidências científicas; incompreensão semântica e pragmática da TC; deficiência nos financiamentos às investigações e a inadequação da comunicação dos resultados de pesquisa.

A comunicação ágil (mais rápido possível) de resultados das investigações científicas, mas sem comprometer o rigor e a integridade da pesquisa, como relatórios de síntese e instruções, é útil e importante às partes envolvidas na $\mathrm{TC}^{37}$. A linguagem da ciência da implementação precisa ser consistente, acessível e compreensível a todos ${ }^{\mathbf{3 8}}$; e para que isso seja alcançado com sucesso, os pesquisadores devem formar laços estreitos com todos as partes interessadas ${ }^{39}$.

Nesse sentido, as Plataformas de TC - uma tipicamente entidade de nível nacional ou estadual projetada para criar e nutrir elos entre pesquisadores, formuladores de políticas e profissionais de saúde - são dispositivos importantes para coleta, avaliação crítica, facilitação e comunicação de evidências ${ }^{27}$. Acredita-se que essa prática ajude os tomadores de decisão a gerenciar o rápido aumento da evidência disponível, agrupando (por meio de uma busca sistemática da literatura disponível), filtrando (identificando fontes confiáveis de informação) e sintetizando (fornecendo uma análise crítica do corpo de evidências), além de determinar (a melhor decisão) e divulgar as provas a todos os potenciais utilizadores em um formato acessível e compreensível ${ }^{40}$.

Outra forma favorável de comunicar resultados de pesquisa e impactar na TC são as fontes tradicionais de mídia, como artigos de televisão e jornais, mas também fontes relativamente novas, como as mídias sociais (blogs, internet e Twitter); isso porque os tomadores de decisão política (por exemplo, políticos) podem sentir que a atitude da mídia tem grande influência sobre os eleitores e, portanto, poderão agir de acordo com o que está sendo publicado nesses setores de comunicação ${ }^{41}$.

Nesse aspecto, os financiamentos de pesquisa precisam incluir a necessidade óbvia de recursos dedicados para apoiar também a troca de conhecimento, além da duração do projeto de pesquisa principal. Portanto, os doadores e financiadores de pesquisa também podem financiar, monitorar e avaliar as atividades de troca de conhecimento, bem como patrocinar o desenvolvimento de revisões sistemáticas, complementando esforços alternativos para melhorar a $\mathrm{TC}^{40}$.

Nossos resultados revelaram que a comunicação clara e de fácil entendimento é primordial para a TC, uma vez que os estudos desta revisão apontam para a inabilidade dos profissionais de saúde em buscar e aplicar as melhores evidências em suas práticas clínicas. Nesses casos, as instituições de saúde necessitam prestar educação continuada e permanente aos seus recursos humanos para o uso de evidências científicas ${ }^{41}$, além de fornecer uma infraestrutura tecnológica de acesso à informação adequada. Além disso, as instituições de pesquisa também podem fornecer treinamentos, focados em melhorar capacidades individuais de comunicação, networking e de compreensão dos contextos científicos e de tomada de decisão ${ }^{\mathbf{3 8}}$.

Entre os pesquisadores, um desafio a ser superado, evidenciado nesta revisão, é o entendimento conceitual da TC. Quando há disjunção semântica, levando a mal-entendidos em torno do que as pessoas estão tentando fazer e esperando conseguir, a ciência da implementação fica comprometida. Assim, as diferenças na interpretação (limite semântico) de TC poderiam ser abordadas por meio de intervenções educacionais; ressaltando que a 
falta de comunicação limita o caminho da implementação de novos conhecimentos, sendo necessário buscar meios para superar nuances semânticas das diferentes interpretações da TC entre pesquisadores e profissionais dos serviços de saúde 39 .

Para transpor essa lacuna entre pesquisadores e público beneficiário da pesquisa, acredita-se que a pesquisa participativa seja um caminho/alternativa. $\mathrm{O}$ avanço da ciência da TC exigirá necessariamente que os mundos da prática e da pesquisa sejam aproximados ${ }^{37}$, porque a pesquisa que tem metas transformadoras precisa ser fundamentada no aqui e agora $^{39}$. No entanto, para isso, faz-se necessário haver o diálogo e o engajamento da parte dos cinco principais setores da sociedade, a saber: organizações de saúde, instituições acadêmicas, centros de pesquisa, organizações governamentais e a comunidade, para garantir que haja alinhamento da atividade de cada um desses grupos (Pesquisa, Educação, Saúde, Governo, Comunidade, [incluindo Indústria]) com o objetivo comum de $\mathrm{TC}^{\mathbf{4 2}}$.

A teorização sobre a implementação não deve, portanto, ser um exercício acadêmico abstrato, desvinculado do mundo real da prática de implementação $0^{43}$. Para implementar uma mudança baseada em evidências de pesquisas, uma nova prática precisaria emergir de dentro do sistema, auto-organizando dos micros (indivíduos) para os macroníveis (subsistemas, estruturas organizacionais) ${ }^{44}$. Isso significa que, ao invés de esperar que as provas sejam produzidas e que, posteriormente, haja engajamento de estruturas de implementação e estratégias de mudança de comportamento para traduzir intervenções complexas em prática clínica, a ênfase deveria idealmente usar estruturas de implementação para entender o contexto, mecanismos e condições anteriores, assim como o modo em que a evidência será ou está sendo gerada ${ }^{45}$.

Os cientistas devem observar e ouvir sobre os problemas que afetam uma comunidade. Pesquisadores e profissionais de saúde devem se engajar em relacionamentos significativos e colaborativos que possam consistir em um grupo de $\mathrm{TC}^{46}$. Interações entre acadêmicos e profissionais de saúde permitem que a experiência clínica forneça conhecimento sobre os problemas relevantes e sobre as necessidades do paciente, bem como sobre os usos das soluções existentes e suas deficiências, já que as unidades clínicas são importantes na fase de geração de ideias, e não apenas em testes e melhorias pós-introdução ${ }^{47}$.

A primeira etapa da fase de projeto de pesquisa, portanto, deve ser a identificação e a articulação de todas as partes interessadas por meio do mapeamento desses grupos ${ }^{38}$, os quais devem ser identificados sistematicamente, o mais cedo possível, idealmente antes da apresentação de propostas de financiamento, para que as partes interessadas possam ajudar a conceber a investigação e a implementação das atividades de $\mathrm{TC}^{37}$.

Um conjunto de princípios a serem implementados rotineiramente como parte de qualquer programa de pesquisa aplicada para a TC inclui: i) mapeamento das partes interessadas antes do início dos programas de pesquisa; ii) perguntas de pesquisa a serem desenvolvidas em conjunto com as partes interessadas; iii) implementação de abordagens de pesquisa participativa; iv) uso de um corretor/mediador de conhecimento; e v) sistemas adaptados de gestão do conhecimento ${ }^{38}$. Embora os corretores/mediadores do conhecimento sejam conceituados e operacionalizados de forma diferente em vários setores, a característica principal desse profissional é facilitar a troca de conhecimento entre os vários interessados ${ }^{38}$.

Dessa forma, pesquisadores e comunidade compartilham o controle do processo de pesquisa e, juntos, identificam problemas, moldam os objetivos da pesquisa, decidem sobre as metodologias, coletam e interpretam os dados, além de disseminar e aplicar as descobertas. Assim, todas as partes interessadas devem ser convidadas para a fase de design para codesenvolver as questões de pesquisa, visando garantir que elas estejam alinhadas com as necessidades de investigação dos grupos ${ }^{\mathbf{3 8}}$. 
Além disso, muito mais atenção deve ser dada ao contexto, aos mecanismos e às condições que levam à geração de resultados de pesquisa, particularmente quando a intervenção for complexa e envolver fatores humanos para o seu sucesso ${ }^{45}$.

O maior número possível de oportunidades para o envolvimento das partes interessadas ao longo do ciclo de pesquisa precisava ser incorporado desde o desenvolvimento de propostas e o planejamento do estudo. Isso porque, para desenvolver uma TC, é essencial promover um sentimento de confiança entre todos os envolvidos, criando um senso de propriedade compartilhada sobre questões de pesquisa e descobertas subsequentes ${ }^{37}$. Para tanto, são necessários acordos que sinalizem os compromissos dessa colaboração, com regras claras e consistentes, como o financiamento de trabalho conjunto, espaço compartilhado, equipamentos e direitos de propriedade intelectual ${ }^{47}$.

Ademais, para o desenvolvimento da TC, deve-se (re)formar o processo de investigação científica, principalmente tentando alinhar tópicos de pesquisa com necessidades políticas, em oposição às necessidades ou desejos dos financiadores. Isto parece especialmente relevante porque todas as partes interessadas (formuladores de políticas, pesquisadores e políticos) precisam realmente demonstrar vontade de cooperar e estar abertas para discutir o uso de evidências na elaboração de políticas ${ }^{41}$. Logo, é imprescindível a construção de confiança entre os formuladores de políticas e pesquisadores. Por essa razão, os projetos podem adaptar melhor seu trabalho para atender às necessidades e prioridades daqueles que têm maior probabilidade de aplicar suas pesquisas em políticas e práticas ${ }^{37}$.

Uma forma de melhorar a troca de conhecimento entre cientistas e tomadores de decisão, bem como fortalecer as relações entre ambos, é a incorporação de cientistas em agências de tomada de decisão ${ }^{40}$. Um trabalho mútuo entre um departamento de governo e uma universidade, em uma ligação formal entre $o$ governo e as organizações de investigação, bem como uma cultura governamental que valorize o uso de pesquisas na formulação de políticas, contribui significativamente para a $\mathrm{TC}^{48}$. Desse modo, percebe-se a importância de ter equipes de ciências inseridas nas agências de tomada de decisão, visando concentrar-se no engajamento mais amplo das necessidades da comunidade ${ }^{\mathbf{3 8}}$.

Vale destacar que pesquisas com abordagens interdisciplinares são muito importantes, pois, além de oportunizar a interação entre acadêmicos, clínicos e formuladores de políticas, elas promovem nos grupos o pensar 'fora da caixa' ${ }^{\mathbf{4 7}}$, ampliando seus olhares para a complexidade da $\mathrm{TC}^{\mathbf{4 2}}$.

O trabalho em rede, com a criação de cooperações intersetoriais, seminários e conferências, em que pesquisadores e formuladores de políticas possam se reunir a fim de facilitar a construção de uma estrutura de referência e linguagem conjunta, é primordial para a $\mathrm{TC}^{\mathbf{3 8 , 4 1}}$. Entretanto, a TC é uma rede complexa composta de sub-redes interdependentes, que interagem dinamicamente de diferentes maneiras em diferentes momentos em um ou mais setores da comunidade, por exemplo: saúde, governo, educação e pesquisa ${ }^{42}$. Destarte, uma coprodução honesta do conhecimento, em uma abordagem de pesquisa participativa que busque a produção e a troca de conhecimentos, pode ser alcançada pela inserção de equipes de ciências nas agências de tomada de decisão, sendo necessário concentrar-se no engajamento mais amplo da comunidade ${ }^{38}$.

Diante disso, é imperativo que os líderes dentro dos sistemas entendam que precisam procurar indivíduos que atuem como eixos, interagindo com outros dentro e entre os grupos $^{\mathbf{4 2}}$, estabelecendo redes entre pesquisadores e tomadores de decisão. Para realizar o elo entre os cientistas e tomadores de decisão, bem como facilitar a troca de conhecimento entre os grupos, é benéfico ter um corretor/ mediador de conhecimento politicamente astuto, com habilidades de comunicação e a capacidade de cultivar redes sociais produtivas e expansivas durante toda a vida do progra$\mathrm{ma}^{38}$. Dessa forma, visa-se construir redes de pesquisa baseadas em práticas, com fortes 
laços de confiança em todo o espectro de $\mathrm{TC}^{\mathbf{4 6}}$. Ressalta-se ainda que os gerentes responsáveis pela inovação em saúde devem garantir que a infraestrutura para colaboração esteja em vigor, em que o elemento central sejam os acordos de estrutura institucional que facilitem a criação de novos empreendimentos cooperativos ${ }^{47}$.

Pelos desafios apontados nesta revisão, observa-se que a ciência da implementação - o estudo científico dos métodos que traduzem os resultados da pesquisa em resultados úteis e práticos - é contestada e complexa ${ }^{39}$. Assim, parece improvável que alguma vez haverá uma teoria de implementação do conhecimento, já que se trata de um fenômeno multifacetado e complexo demais para permitir explicações universais ${ }^{\mathbf{4 3}}$.

O desafio permanece em torno de como o conhecimento se mobiliza entre as partes interessadas; e, neste caso, propõe-se que o espaço a ser percorrido não seja caracterizado por lacunas, mas por sinapses de interação e conectividade. Então, é de vital importância promover a oportunidade de engajamento precoce dos setores para que essas conexões ocorram ${ }^{42}$. Esse engajamento para reduzir a brecha entre as evidências geradas e a prática clínica precisa acontecer ainda como pensamento prévio dos desenhos de pesquisa ${ }^{45}$. Além disso, deve haver clareza sobre os resultados pretendidos e a necessidade de contínua comunicação e interpretação dos dados pelas partes interessadas, buscando mutualmente a compreensão de quaisquer suposições sobre os motivos pelos quais as atividades específicas produziriam esses resultados ${ }^{37}$.

No entanto, devido a restrições econômicas e logísticas, esse acoplamento pode nem sempre ser prático, requerendo formas alternativas de combinar pesquisa e prática, como uma metodologia de pesquisa qualitativa, por meio de entrevistas individuais e grupos focais ${ }^{49}$. Outrossim, seria importante a realização de uma pesquisa empírica para estudar como e até que ponto o uso de teorias, modelos e estruturas de TC contribui para uma implementação mais efetiva dos resultados de investigações e sob quais condições ou circunstâncias contextuais eles se aplicam ou não ${ }^{\mathbf{4 3}}$.

Por fim, é mister pontuar a necessidade de concentrar-se no engajamento mais amplo da comunidade, porque o envolvimento da comunidade na ciência pode levar ao estabelecimento de licenças sociais e aceitação de ações de gestão em uma ciência cidadã, por meio dos quais os membros da comunidade participem ativamente dos programas de pesquisa científica, como um meio de envolvimento da comunidade na ciência ${ }^{38}$. Cabe destacar que TC está dentro do paradigma social construtivista, pois privilegia a interação social e a adaptação de evidências de pesquisa que levam em consideração o contexto e a cultura locais, em uma visão holística dos fenômenos, integrando os conceitos de criação e ação do conhecimento ${ }^{50}$.

\section{Limitações do estudo}

Embora já se tenha tentado desenvolver uma estratégia de busca abrangente, é possível que alguns estudos relevantes tenham sido perdidos. Igualmente, materiais e pesquisas que não foram publicados em periódicos acadêmicos nas bases de dados PubMed, Scopus e Web of Science, como documentos governamentais ou anais de eventos científicos, não foram pesquisados e poderiam ter fornecido algumas informações adicionais. Além disso, o tema Prática Baseada em Evidência (PBE) não foi considerado na busca desta revisão, apesar de ser aceito por muitos estudiosos como sinônimo de TC. Assim, somente artigos que abordam especificamente TC foram selecionados nesta revisão de escopo.

Ao contrário das revisões sistemáticas, as revisões de escopo não incorporam uma avaliação de qualidade dos estudos para sua inclusão. No caso, os estudos incluídos nesta revisão não foram avaliados por seu rigor científico, pois, como já dito, as revisões de 
escopo geralmente não incluem avaliações críticas das metodologias empregadas nos estudos. Ao decidir resumir e relatar as descobertas gerais sem o escrutínio de um processo de avaliação formal, reconhece-se que se pode ter negligenciado alguns aspectos dos desafios da TC apresentados nesta revisão.

Outra limitação potencial é que pesquisando apenas os títulos, resumos e títulos de assunto, pode-se ter perdido documentos relevantes. Além disso, a seleção dos estudos foi realizada somente por um autor, o que pode limitar a confiabilidade dos resultados. Por fim, ressalta-se que algumas dessas características metodológicas são comuns em revisões de escopo.

\section{Considerações finais}

Esta revisão de escopo identificou que, em todo o processo da TC, há desafios, e eles estão presentes desde a produção de um novo conhecimento até a sua implementação nas práticas clínicas de cuidados à saúde. Um dos principais desafios é minimizar a assimetria entre o universo da pesquisa e as práticas em saúde. Outro desafio para a TC é a falta de financiamentos em pesquisa que promovam não somente a produção do conhecimento (investigações primárias), mas também a sua implementação.

Além disso, a comunicação deficitária dos resultados de investigações, por falta de praticidade - com interpretações estatísticas de difícil compreensão e a ausência de descrições de estratégias de implementação de novos conhecimentos -, também é um desafio a ser superado.

Igualmente, a falta de apoio das instituições/organizações de saúde em promover capacitações, espaços e infraestrutura para a implementação de novas evidências científicas constitui-se em um desafio à TC que precisa ser transposto. Ainda com relação às instituições, outro desafio é a falta de habilidade dos profissionais de saúde em buscar, selecionar e aplicar novos conhecimentos. Essa falta de domínio científico desses profissionais gera uma limitação importante para a TC.

Com vista a mitigar os obstáculos, suplantando os desafios da TC na área da saúde, propõe-se:

- Criar redes de pesquisa, com participação da comunidade acadêmica, serviços de saúde, representantes comunitários (grupos de pacientes) e governamentais;

- Ampliar os financiamentos de pesquisa para além da etapa de produção de novos resultados, disponibilizando assim recursos que possibilitem a aplicação e a avaliação de novas tecnologias e práticas em saúde;

- Produzir relatórios de investigação com linguagem acessível ao público consumidor (profissionais de saúde e gestores, principalmente), contendo também propostas de implementação dos resultados encontrados;

- Promover, pelas instituições de saúde, capacitações de recursos humanos e de processos organizacionais de trabalho - como tempo e tecnologias - para o desenvolvimento da TC;

- Desenvolver habilidades e competências em investigações científicas durante a formação profissional na área da saúde, para que os novos formandos ingressem no mercado de trabalho com aptidão para desenvolver e consumir pesquisas.

Em conclusão, superar os desafios contemporâneos da TC vai além de um movimento único, pois é preciso mobilizar todos os setores responsáveis por produzir e implementar ações em saúde. Nesse sentido, faz-se necessário fortalecer redes, com nós consistentes, entre pesquisadores, profissionais de saúde (que conhecem as demandas dos pacientes e serviços) e tomadores de decisão política. Igualmente, essa rede não pode negligenciar o espaço e o poder que a sociedade civil tem nela, pois movimentos populares mobilizam políticas e políticos. 


\section{Colaboradores}

Ferraz L (0000-0002-2487-8614)* contribuiu para a concepção e o planejamento do estudo, para a coleta, análise e a interpretação dos dados, elaboração do artigo, aprovação da versão final do manuscrito. Pereira RPG
(0000-0002-4811-6753)* e Pereira AMRC (0000-0001-8467-6398)* contribuíram para a concepção e o planejamento do estudo, análise e a interpretação de dados, revisão crítica do conteúdo e aprovação da versão final do manuscrito.

\section{Referências}

1. Pablos-Mendez A, Shademani R. Knowledge translation in global health. J. contin. educ. health prof. 2006; 26:81-86.

2. Canadian Institutes of Health Research (CIHR). Knowledge Translation Strategy 2004-2009: Innovation in Action. Ottawa: CIHR, 2004. [acesso em 2018 abr 20]. Disponível em: http://www.cihr-irsc.gc.ca/e/26574. html.

3. World Health Organization. Bridgingthe "Know-Do" gap: reporton meeting on knowledge translation in global health. Geneva: WHO; 2006. [acesso em 2018 abr 20]. Disponível em: https://www.measureevaluation.org/resources/training/capacity-building-resources/high-impact-research-training-curricula/ bridging-the-know-do-gap.pdf.

4. Davison CM. Knowledge translation: implications for evaluation. In: Ottoson JM, Hawe P, organizadores. Knowledge utilization, diffusion, implementation, transfer, and translation: implications for evaluation. Washington: American Evaluation Association; 2009. p. 75-87.

5. Reimer-Kirkham S, Varcoe C, Browne AJ, et al. Critical inquiry and knowledge translation: exploring compatibilities and tensions. Nurs. philos. and Contributor ID).

2009, 3:152-66.
6. Grimshaw J, Eccles M, Lavia J, et al. Knowledge translation of research findings. Implement. sci. 2012; 50:117.

7. Rapport F, Clay-Williams R, Churruca K, et al. The struggle of translating science into action: foundational concepts of implementation science. J. eval. clin. pract. (Online). 2018; 24(1):117-126.

8. Oborn E, Barrett M, Racko G. Knowledge translation in healthcare: a review of the literature. Cambridge: Cambridge Judge Business School; 2010.

9. Aromataris E, Munn Z, editores. Joanna Briggs Institute Reviewer's Manual. The Joanna Briggs Institute, 2017. [acesso em 2018 abr 20]. Disponível em: https://reviewersmanual.joannabriggs.org/.

10. Levac D, Colquhoun H, O'Brien KK. Scoping studies: advancing the methodology. Implement. sci. 2010; 5(1):5-69.

11. Colquhoun HL, Levac D, O’Brien KK, et al. Scoping reviews: time for clarity in definition, methods, and reporting. J. clin. epidemiol. 2014; 67(12):1291-1294.

12. Joanna Briggs. Institute. The Joanna Briggs Institute reviewers' manual 2015: Methodology for JBI scoping reviews; 2015. [acesso em 2018 abr 20]. Disponí- 
vel em: http://joannabriggs.org/assets/docs/sumari/ Reviewers-Manual_Methodology-for-JBI-Scoping-Reviews_2015_v2.pdf.

13. Tricco AC, Antony J, Soobiah C, et al. Knowledge synthesis methods for generating or refining theory: a scoping review reveals that little guidance is available. J. clin. Epidemiol. 2016; 73:36-42.

14. Arksey H, O'Malley L. Scoping studies: towards a methodological framework. Int. j. soc. res. methodol. 2005; 8:19-32.

15. Peters MDJ, Godfrey C, McInerney P, et al. Scoping Reviews. In: Aromataris E, Munn Z, editores. Joanna Briggs Institute Reviewer's Manual. Australia: The Joanna Briggs Institute; 2017.

16. Welch V, Petticrew M, Petkovic J, et al. Extending the PRISMA statement to equity-focused systematic reviews (PRISMA-E 2012): explanation and elaboration. J. clin. epidemiol. 2016; 70:68-89.

17. Metzler MJ, Metz GA. Analyzing the barriers and supports of knowledge translation using the PEO model. Can J Occup Ther. 2010; 77(3):151-158.

18. Oelke ND, Lima MAS, Acosta AM. Knowledge translation: translating research into policy and practice. Rev. gaúch. enferm. 2015; 36(3):113-117.

19. Nabyonga OJ, Marchal B, Mafigiri D, et al. Perspectives on the role of stakeholders in knowledge translation in health policy development in Uganda. BMC health serv. res. (Online). 2013; 13:324.

20. Menear M, Grindrod K, Clouston K, et al. Advancing knowledge translation in primary care. Can. fam. physician. 2012; 58(6):623-627.

21. Azimi A, Fattahi R, Asadi-Lari M. Knowledge translation status and barriers. J. med. libr. assoc. 2015; 103(2):96-99.

22. Muntaner C, Chung H, Murphy K. Barriers to knowledge production, knowledge translation, and urban health policy change: ideological, economic, and poli- tical considerations. J. urban health. 2012; 89(6):915-

924.

23. Hu G, Sun Z. Poor knowledge translation: an urgent problem in China. Lancet. 2008; 372(9640):718.

24. Nedjat S, Gholami J, Yazdizadeh B, et al. Research's Practice and Barriers of Knowledge Translation in Iran. Iran. j. public health. 2014; 43(7):968-980.

25. Liu W, Shi L, Pong RW, et al. Determinants of knowledge translation from health technology assessment to policy-making in China: from the perspective of researchers. PLoS One. 2018; 13(1):1-13.

26. Wehrens R. Beyond two communities - from research utilization and knowledge translation taco-production? Public health. 2014; 128(6):545-551.

27. Kasonde JM, Campbell S. Creating a Knowledge Translation Platform: nine lessons from the Zambia Forum for Health Research. Health res. policy syst. 2012; 10(31):1-8.

28. Murphy K, Fafard P. Takingpower, politics, and policy problems seriously: the limits of knowledge translation for urban health research. J. urban health. 2012; 89(4):723-732.

29. Visram S, Goodall D, Steven A. Exploring conceptualizations of knowledge translation, transfer and exchange a cross public health in one UK region: a qualitative mapping study. Public health. 2014; 128(6):497-503.

30. Liu W, Shi L, Pong RW, et al. Differences in evaluating health technology assessment knowledge translation by researchers and policymakers in China. Int. j. technol. assess. health care. 2014; 30(6):612-620.

31. Bartlett G, Gagnon J. Physicians and knowledge translation of statistics: mind the gap. CMAJ. 2016; 188(1):11-12.

32. Engebretsen E, Sandset TJ, Ødemark J. Expanding the knowledge translation metaphor. Health res. policy syst. 2017; 15:19. 
33. Curran JA, Grimshaw JM, Hayden JA, et al. "Knowledge translation research: the science of moving research into policy and practice”. J Contin Educ Health Prof. 2011; 31(3):174-180.

34. Harvey G, Marshall RJ, Jordan Z, et al. Exploring the Hidden Barriers in Knowledge Translation: a Case Study Withinan Academic Community. Qual. health res. $2015 ; 25(11): 1506-1517$.

35. Bengough T, Bovet E, Bécherraz C, et al. Swiss family physicians' perceptions and attitudes towards knowledge translation practices. BMC. 2015; 16:177.

36. Bigham BL, Aufderheide TP, Davis DP, et al. Knowledge translation in emergency medical services: a qualitative survey of barriers to guideline implementation. Resuscitation. 2010; 81(7):836-840.

37. Reed MS, Stringer LC, Fazey I, et al. Five principles for the practice of knowledge exchange in environmental management. J. environ. manag. 2014; 146:337345 .

38. Cvitanovic C, McDonald J, Hobday AJ. From science to action: principles for undertaking environmental research that enables knowledge exchange and evidence-based decision-making. J. environ. manag. 2016; 183(3):864-874.

39. Rapport F, Clay-Williams R, Churruca K, et al. The struggle of translating science into action: Foundational concepts of implementation science. J. eval. clin. pract. 2018; 24(1):117-126.

40. Cvitanovic C, Hobday AJ, Van Kerkhoff L, et al. Improving knowledge exchange among scientists and decision-makers to facility ate the adaptive governance of marine resources: a review of knowledge e and research needs. Ocean \& Coastal Management. 2015; (112):25-35.

41. Van de Goor I, Hämäläinen RM, Syed A, et al. Determinants of evidence use in public health policymaking: results from a study across six EU countries. Health policy. 2017; 121(3):273-281.
42. Kitson A, Brook A, Harvey G, et al. Using Complexity and Network Concepts to Inform Healthcare Knowledge Translation. Int J Health Policy Manag. 2017; $7(3): 231-243$.

43. Nilsen P. Making sense of implementation on theories, models and frameworks. Implement Sci. 2015; (21):10:53.

44. Chandler J, Rycroft-Malone J, Hawkes C, et al. Application of simplified complexity theory concepts for health care social systems to explain the implementation of evidence into practice. J. adv. nurs. 2016; $72(2): 461-480$.

45. Brocklehurst PR, Williams L, Burton C, et al. Implementation and trial evidence: a plea for fore-thought. BDJ. 2017; 222:331-335.

46. Eisenmann JC. Translational gap between laboratory and playing field: New era to solve old problems in sports science. TJACSM. 2017; 2(8):37-43.

47. Thune TM, Gulbrandsen M. Combining knowledge to generate novelty: a study of disclosed ideas for life science inventions. Eur J Innov Manag. 2017; 20(3):446-462.

48. O’Donoughue JL, Anstey KJ. The use of secondments as a tool to increase knowledge translation. Public Health Res. Pract. 2017; 27(1):1-4.

49. Harper LD, McCunn R. Hand in Glove: Using Qualitative Methods to Connect Research and Practice. IJSPP. 2017; 12(7):990-993.

50. Graham ID, Tetroe JM. The Knowledge to Action framework. In: Rycroft-Malone J, Bucknell T, editores. Models and Frameworks for Implementing Evidence-Based Practice: linking evidence to action. Oxford: Wiley-Blackwell; 2010. p. 207-222.

\footnotetext{
Recebido em 09/03/2019

Aprovado em 24/08/2019

Conflito de interesses: inexistente

Suporte financeiro: não houve
} 